\title{
Producción de textos y desarrollo de la conciencia ambiental en estudiantes de educación primaria
}

\author{
Gabriel Abraham Díaz Flores \\ gdiazfl@ucvvirtual.edu.pe \\ Marilyn Melisa Ardiles Azaña \\ marilyn_01_01@hotmail.com \\ Groberti Alfredo Medina Corcuera \\ grome@ucvvirtual.edu.pe \\ Universidad César Vallejo \\ Chimbote - Perú
}

\section{RESUMEN}

El estudio tuvo como objetivo general determinar que el programa produciendo textos, desarrolla la conciencia ambiental en los estudiantes del IV ciclo de educación primaria de la IE $N^{\circ}$ 88027, Lacramarca Baja, 2021. La metodología empleada considera la investigación tipo aplicada con enfoque cuantitativo, método hipotético-deductivo y con diseño pre experimental. La muestra estuvo constituida por 30 estudiantes del $3^{\circ}$ y $4^{\circ}$ grado de educación primaria, pertenecientes al IV ciclo. Se empleó la técnica de la encuesta y como instrumento de recolección de datos un cuestionario para evaluar la conciencia ambiental, validado por 2 jueces expertos y con una confiabilidad de 0,71 obtenido a través del Alfa de Cronbach. Los resultados obtenidos demuestran un incremento de $43 \%$ en el nivel bueno y $44 \%$ en el nivel muy bueno, asimismo, a través de la prueba estadística de Wilcoxon se determinó el valor de $\mathrm{Z}=-4.800$ menor a -1.96 y Sig. $=0.000$ menor a 0.05 , rechazando la $\mathrm{H}_{0}$ y aceptando la hipótesis del investigador. Concluyéndose que la aplicación del programa produciendo textos influyó de manera significativa en el desarrollo de la conciencia ambiental de los estudiantes del IV ciclo de educación primaria de la IE $\mathrm{N}^{\circ} 88027$.

Palabras clave: conciencia ambiental; dimensión cognitiva; dimensión afectiva; dimensión conativa; dimensión activa 


\title{
Producing texts and development of environmental awareness in elementary students
}

\begin{abstract}
The purpose of the study was to determine that the program of production text, develops environmental awareness in students of the IV cycle of primary education of the I.E 88027, Lacramarca Baja, 2021. The methodology used takes into account the type of research applied with a quantitative approach, a hypothetical-inferential method and a pre-experimental design. The sample consisted of 30 pupils at the $3^{\circ}$ and $4^{\circ}$ levels of primary education, belonging to the IV cycle. The survey technique was used and as a data collection tool a questionnaire to evaluate environmental awareness, validated by 2 expert judges and with a reliability of 0.71 obtained through the Cronbach Alpha. The results show an increase of $43 \%$ in the good level and $44 \%$ in the very good level, also through the Wilcoxon statistical test the value of $\mathrm{Z}=-4,800$ less to -1.96 and Sig. $=0,000$ lower than 0,05 , rejecting $\mathrm{H}_{0}$ and accepting the hypothesis of the scientist. Conclusion that the implementation of the text generation program has significantly affected the development of environmental awareness at I.E. N ${ }^{o} .88027$ IV primary school students.
\end{abstract}

Keywords: environmental awareness; cognitive dimensión; affective dimensión; conative dimensión; active dimensión

Artículo recibido: 02 noviembre. 2021 Aceptado para publicación: 28 noviembre 2021 Correspondencia: gabriel_arga9@ hotmail.com

Conflictos de Interés: Ninguna que declarar 


\section{INTRODUCCIÓN}

A nivel mundial, atravesamos esta pandemia del COVID-19, tomando conciencia sobre nuestro accionar con el medio ambiente. Ante este contexto, la contaminación ha sido parte de la afectación de nuestra salud (Banco Mundial, 2020). Debemos tener en cuenta, cuando iniciamos un emprendimiento, el impacto de nuestras acciones con el medio del cual somos parte, tomar en cuenta los diversos niveles donde impacta este accionar humano (Quispe, et al., 2020). Castillo, Ruíz y Flores (2018), señalaron que, no existe una adecuada prevención para que se detenga el desarrollo de los problemas ambientales. Para tener un mayor conocimiento de la afectación de esta situación, la Organización Mundial de la Salud (OMS, 2018), sostuvo en torno a 12 millones de individuos al año dejan de vivir como consecuencia de los riesgos ambientales. Lo cual se deduce que existe una escasa conciencia sobre la importancia del medio ambiente.

Los problemas ambientales ocurren frecuentemente con países en desarrollo y vinculados con el impulso de la industria, pero que se eximen de las responsabilidades con el ambiente (Rathee y Thakran (2017), evidencia clara, del bajo nivel de conciencia ambiental. Sin embargo, en ciertos países tratan de comprender mejor el desarrollo sostenible. Por ejemplo, en Colombia, el gobierno, dispuso disminuir hasta el 2030 un $51 \%$ los gases de efecto invernadero (Revista Semana, 2021). En Ecuador, se declaró la necesidad de establecer políticas enfocadas a la educación ambiental (Falconí, Reinoso, Collado, Hidalgo y León 2019). Estas situaciones impulsan un desarrollo de conciencia ambiental.

A nivel nacional, el Perú no es ajeno al tema ambiental, el Instituto Nacional de Estadística e informática (INEI, 2021), con relación sobre los fenómenos antrópicos, evidenció una variación porcentual muy elevado en el 2021 con el 271,4\% respecto del año 2020. Un dato que relaciona al país incluido en el puesto 21 entre los más contaminados (Ospina, 2019). La nulidad de conciencia ambiental traslada a una ausencia de involucramiento de individuos, en la proyección de los temas ambientales (Laso, Marban y Ruíz, 2017). Asimismo, existe un mayor porcentaje de conciencia ambiental en individuos de zonas rurales que de zonas urbanas (Punzalan, Signo, Mae, Signo, y Marasigan, 2019), y este tema no debe ser indistinto para nadie. Ni existir, diferencia alguna por género (Nagihan, 2020). Ante lo señalado, Yıldız (2019), menciona la necesidad de tener algunas habilidades básicas para comprender el desarrollo de la naturaleza y su vinculación con el hombre. González y Meira (2020), rescataron que el 
cambio ambiental compone el reto más relevante del hombre en el contexto presente. Además, es necesario que se replique de manera considerable la previsión por el medio ambiente y de manera oportuna a través del currículo (Eliseo, 2020).

A nivel regional, Áncash está inmersa en esta situación. Una de las estrategias que se propone para mejorar la interacción entre el medio y el hombre es el diálogo reflexivo (Santacruz, 2018). Para Flores, Gómez, y Osman (2017), es indudable que el desarrollo de la sociedad necesite de las interacciones de todos sus entes. Muchas veces, miramos desde lejos los acontecimientos y debe marcarse como erróneo ya que, según Moyano (2018), todos formamos parte de un mismo lugar. Existe un problema al momento de interiorizar la correspondencia armónica del ser humano con el ambiente, y es, sin lugar a duda, en la etapa de la niñez donde deben aprender este propósito (Kabadayi y Altinsoy, 2019). Se debe buscar la relación entre el impacto de la contaminación y los individuos para acercarnos a la conciencia ambiental (Chierrito, Martins, De Souza, Da Silva y Grossi, 2018). Del mismo modo, debe implicar la participación activa para mejorar la gestión ambiental (León et al., 2018).

Muchos de los estudiantes aducen conocer sobre el medio ambiente, sin embargo, poseen escasos conocimientos (Gervacio y Castillo, 2020). Ante esto, se pueden aplicar diversos programas para mejorar, como las que se aplican en ingeniería (Vargas, 2021), sin embargo, estos se ven obstaculizados por la no disposición al cambio (Aliman, Budijanto, Sumarmi y Astina, 2019). Además, se necesita el involucramiento de la familia en cuanto al crecimiento de conciencia ambiental en los estudiantes (Yeşilyurt, Özdemir, y Erol, 2020).

Dentro de las aulas, se debe promover la educación ambiental como parte fundamental de la vida (Aparecida, Siqueira y De Cássia, 2018). Para Emine (2019), la influencia de los docentes en la concientización ambiental, es muy imprescindible ante los educandos puesto que les facilita desarrollar una susceptibilidad con el problema ambiental. En el $3^{\circ}$ y $4^{\circ}$ de educación primaria en la IE $N^{\circ} 88027$, hubo una problemática con respecto a la conciencia ambiental, evidenciada por medio de la observación sistemática sobre la práctica ambiental. Ante lo mencionado, quedó expuesto la necesidad de aplicar un programa en beneficio de la conciencia ambiental para los estudiantes. Se intenta mejorar la educación con nuevas implementaciones y recursos (Susilawati, Aznam, Irwanto y Paidi, 2020). 
Ante la necesidad de querer revertir los datos negativos presentados, se formuló el problema: ¿En qué medida el programa produciendo textos desarrolla la conciencia ambiental en estudiantes de educación primaria de la IE $\mathrm{N}^{\circ} 88027$, Lacramarca Baja 2021? Se justifica porque aporta al conocimiento de la aplicación del programa, como parte del proceso de enseñanza-aprendizaje. Asimismo, nace como respuesta a la coyuntura actual que estamos viviendo, para contribuir con las buenas prácticas ambientales y encaminar la conciencia ambiental. Asimismo, sirve como base para futuras investigaciones sobre la exploración de la misma variable en otros contextos. Por otro lado, se creó un instrumento, válido y confiable, que cuantifica el nivel de conciencia ambiental y, puede ser empleado como pilar en la medición para esta variable.

El objetivo general fue determinar que el programa produciendo textos, desarrolla la conciencia ambiental en estudiantes. Como objetivos específicos se presentó el establecer la influencia del programa produciendo textos en las dimensiones cognitiva, afectiva, conativa y activa de la conciencia ambiental, así como, elaborar la propuesta de un programa para desarrollar conciencia ambiental. Considerando como hipótesis de investigación: el programa produciendo textos, desarrolla significativamente la conciencia ambiental, y como hipótesis nula: El programa produciendo textos, no desarrolla la conciencia ambiental.

Con respeto a los trabajos previos, se tomó en cuenta a Gómez (2017), quien en su tesis referido al fomento de la conciencia ambiental a partir de un programa experimental en educación primaria, expuso que existe una influencia significativa en cuanto a la aplicación del programa propuesto en la investigación y en cuanto a las dimensiones presentadas en esta investigación sobre la conciencia ambiental, se determinó que fueron influidos positivamente a través de la aplicación del programa; conocimiento ambiental, posicionamiento ambiental y conducta ambiental. Rueda (2017), en su tesis, fomento de la conciencia ambiental a través del programa en educación ambiental, determinó que existe carencia en cuanto a la formación de conciencia ambiental en la institución educativa $\mathrm{N}^{\circ} 10214$ “Abel Ramos Purihuamán” ya que no se han logrado aplicar propuestas algunas para desarrollar la conciencia ambiental en cada uno de los entes de la comunidad educativa. De acuerdo a la falta de conciencia ambiental observada se procedió a diseñar y aplicar una propuesta en Educación Ambiental, teniendo como finalidad mejorar el ambiente en la comunidad. Salazar (2017), en su tesis desarrollo de la conciencia ambiental por medio de la aplicación del programa "Educamp" en 
estudiantes de educación primaria, destacó que antes de aplicar el programa se observaron, tanto el grupo experimental y el grupo control similares niveles de conciencia ambiental, además señaló que el resultado del post-test es 9.98, indicando que luego de ejecutar el Programa "Educamp" los estudiantes pertenecientes al grupo experimental y control manifiestan diferencia significativa en la conciencia ambiental, rescatando que el primer grupo desarrolló más conciencia ambiental que el grupo control. Ruiz (2019), en su tesis referido al desarrollo de la conciencia ambiental a través del programa "Mi Mundo Verde”, destacó que con la aplicación del programa prevista en la investigación se ha permitido que los estudiantes del $3^{\circ}$ de primaria mejoren la conciencia ambiental en niveles buenos y muy buenos, determinado con una significatividad Sig. < 0,05. Guerrero (2020), en su tesis referida al desarrollo de conciencia ambiental a través de una propuesta de organización de residuos sólidos, que el 72\% de estudiantes del V ciclo de educación primaria presentan niveles de inicio y proceso, respecto de su conciencia ambiental. Iglesias (2021), en su tesis sobre conciencia ambiental y la propuesta Ambienti Kids aplicado a niños de $3^{\circ}$ primaria, destacó un nivel de significancia $\mathrm{p}=0,000$, denotando significatividad en la influencia del programa sobre el desarrollo de conciencia ambiental. Suarez (2021), en su tesis, mejora de la conciencia ambiental en el nivel secundaria a partir del programa "Dejando huellas verdes", determinó la significatividad de la aplicación del programa, potenciando las dimensiones cognitiva, afectiva, conativa, y activa en estudiantes del nivel secundario. Palma (2020), en su tesis, referida a un estudio desde una perspectiva pedagógica, sobre los docentes en educación básica regular y la conciencia ecológica, destacó que en los distintos niveles de educación existe una insuficiente formación de conciencia ecológica, siendo los docentes responsables de debilitar estas acciones negativas.

Asimismo, en lo que respecta a las bases teóricas consignadas nos apoyamos en Du et al. (2018), quienes nos señalan que la conciencia ambiental es la capacidad de interiorizar las interrelaciones que se dan a partir de la situación real de la calidad del ambiente, el desarrollo de las actividades del hombre y la intención que se tiene de ser partícipe en las actividades del medio ambiente. Es decir, tomar conciencia de los problemas ambientales (Avellaneda, González y Sáez, 2018). Para Nazarenko y Kolesnik (2018), la conciencia ambiental es la línea por la cual se da la trascendencia de la competencia ambiental, centrándose en los estudiantes. Esto apunta a un desarrollo oportuno de la conciencia ambiental enmarcada dentro de los principios de la educación, buscando un progreso de 
la calidad de vida en las personas. Con respecto a la dimensión cognitiva, Laso, Marbán y Ruiz (2019), consideran que la conciencia ambiental tiene una extremada relación con la obtención de información. es la agrupación de conocimientos sobre el medio ambiente y su problemática, determinar la información exacta con respecto a lo que se está buscando y emitir un juicio a partir de ello. En referencia a la dimensión afectiva, Mediavilla, Medina y González (2020), señalan que es la asociación de sentimientos y emociones involucradas directamente con la problemática ambiental, dirigiendo no solo su preocupación al medio en el cual coexisten, sino también, cómo perciben lo que los rodea, implicando la integración de valores. Podemos señalar que la dimensión afectiva se introduce en la persona como forma de evaluación sobre su preocupación de los problemas ambientales, desplegando una serie de sentimientos y emociones con respecto al ambiente y los valores ambientales. Con respecto a la dimensión conativa, siguiendo a López (2020), esta dimensión abarca las propias actitudes encaminadas por los sentimientos y lo concerniente a su compromiso con el medio ambiente del cual es parte, asimismo, asumir con compromiso las contemplaciones dentro del marco de la política ambiental. En referencia a la dimensión activa, López (2020), señala que es una práctica que llega a ser realizada de manera voluntaria mirando el objetivo que es la mejora y/o cuidado del medio ambiente, teniendo en cuenta los aprendizajes adquiridos para identificar las razones de los problemas y formular opciones para solucionar estos problemas ambientales. Podemos rescatar, que la dimensión activa es el desarrollo de toda práctica individual y colectiva, así como la promoción de comportamientos responsables dentro del marco ambiental.

Con relación a la variable producción de textos, el Minedu (2017) hace referencia sobre la composición de un sentido de lo que se pretende comunicar, tratándose de un proceso de reflexión. Es una competencia comunicativa, teniendo en cuenta lo que queremos trasmitir, reflejada en un escenario concreto. Respecto de la dimensión planificación, Vine (2020), la posesiona como un proceso en la cual se tienen que reflexionar antes de plasmar la escritura ya que, en muchos casos, esta etapa se ve omitida por considerarla innecesaria dentro de la producción de textos, trayendo como consecuencia la desorganización del texto escrito. Comprende a una anteposición de ideas, información y recursos que se dispone hasta ese momento para empezar a organizar y desarrollar el proceso de escritura respecto a la información contextualizada que se quiere trasmitir. Con respecto a la dimensión textualización, el Minedu (2017) señala que este proceso 
plasma las ideas ordenadamente en un lenguaje escrito, involucrando un orden lógico, acentuándose de manera coherente y cohesionada. Se señala entones que la textualización es la acción propia de registrar de manera escrita, en un papel u otro recurso, lo previsto en la planificación, teniendo en cuenta la sintaxis, los recursos ortográficos y la organización del contenido. En referencia a la dimensión de revisión Di Alessio y Destefanis (2017), asumen que quien escribe tiene la obligación de ir monitoreando lo que plasma y ajustar lo que escribe, de tal manera que incorpore las correcciones necesarias y oportunas releyendo o las que se le proporcionen otros lectores. Podemos señalar que la revisión consiste en perfeccionar el producto final de la textualización, poniéndose en marcha la reflexión de lo que en un inicio se planificó, como la intención de su escrito, las reglas ortográficas, las relaciones lógicas entre párrafos, determinadas por la coherencia y cohesión, desajustes que deben ser subsanados en esta etapa. En relación a la dimensión publicación, el Minedu (2015) señala que es un proceso en el cual se da la socialización de las producciones, adicionando que se deben concertar distintos tipos de publicación, como los murales, elaboración de un libro con las producciones, carteles publicitarios, entre otros. En consecuencia, a lo mencionado, con la publicación, como estrategia didáctica, las producciones deben llegar al destinatario, ya sean dentro o fuera de la institución educativa.

\section{ESTRATEGIAS METODOLÓGICAS O MATERIALES Y MÉTODOS}

Se manejó el tipo de investigación aplicada, empleándose el diseño preexperimental, adaptándole una propuesta al resultado de la investigación, considerando el trabajo con un grupo único, aislando la comparación de grupos.

\section{GE: $\quad O_{1}$}

\section{Donde:}

$$
\begin{array}{ll}
\mathrm{GE} & : \text { Grupo experimental } \\
\mathrm{O}_{1}-\mathrm{O}_{2} & : \text { Pre test }- \text { Pos test } \\
\mathrm{X} & : \text { Variable experimental } \\
\mathrm{P} & : \text { Propuesta }
\end{array}
$$


La ruta posible para resolver el problema que se presentó, estuvo enmarcada por el enfoque cuantitativo, asumiendo el método hipotético-deductivo. La población estuvo conformada por todos los estudiantes de los tres niveles de educación (169 estudiantes), de la Institución Educativa $\mathrm{N}^{\circ}$ 88027, del Centro Poblado de Lacramarca Baja. Determinando una muestra por conveniencia a 31 estudiantes de $3^{\circ}$ y $4^{\circ}$ grado de Educación Primaria, correspondiente al IV ciclo de la Educación Básica Regular. La técnica empleada fue la encuesta y se utilizó el instrumento cuestionario, diseñado por el investigador, aplicándose como pre y post test.

La investigación se desarrolló inicialmente con la planificación e implementación del Programa produciendo textos y la formulación del instrumento de evaluación (test). Se continuó con los procesos de validación y confiabilidad del instrumento (cuestionario pre y post test) de manera oportuna y responsable. Seguidamente, se promovió la gestión administrativa, solicitando el permiso correspondiente a la institución educativa para la ejecución del programa y el instrumento, lográndose recepcionar, de manera formal, una respuesta afirmativa. De inmediato se desarrolló una reunión con los estudiantes y sus padres, participantes del programa, para hacer de su conocimiento, coordinar su aplicación y absolver algunas dudas ya que se desarrolló de manera remota. Luego de ello, se procedió con la aplicación del pre test al grupo de estudio, a través de la plataforma Zoom. Posterior a ello, se desarrolló e implementó el Programa produciendo textos, considerando 20 sesiones de aprendizaje durante dos meses aproximadamente. Culminada las sesiones se dio inicio a la aplicación del post test, para recoger información acerca de la influencia del programa presentado sobre el desarrollo de la conciencia ambiental. Acto seguido, se desarrolló el análisis de datos, empleando la estadística descriptiva e inferencial. Con respecto a la estadística descriptiva, para el procesamiento y análisis de los datos se hizo uso del programa Excel 2019 y SPSS 25, obteniéndose tablas estadísticas, sistematizado la información recopilada, mientras que, en referencia a la estadística inferencial se llegó a efectuar la prueba de normalidad de los datos, aplicando el test de Shapiro Wilk, por contar con una muestra inferior a 50 estudiantes, obteniéndose una distribución no paramétrica, para el cual resultó necesario emplear la prueba estadística de Wilcoxon para muestras relacionadas, concluyendo con la aceptación de la hipótesis de investigación. 


\section{RESULTADOS Y DISCUSIÓN}

\subsection{Análisis descriptivo}

\section{Tabla 1}

Nivel de Conciencia Ambiental de estudiantes del IV ciclo de primaria de la IE $N^{\circ} 88287$

\begin{tabular}{lcccc}
\hline \multirow{2}{*}{ Nivel } & \multicolumn{2}{c}{ Pre Test } & \multicolumn{2}{c}{ Post Test } \\
\cline { 2 - 5 } & Fi & \% & fi & \% \\
\hline Malo & 17 & $57 \%$ & 0 & $0 \%$ \\
Regular & 10 & $33 \%$ & 1 & $3 \%$ \\
Bueno & 2 & $7 \%$ & 15 & $50 \%$ \\
Muy bueno & 1 & $3 \%$ & 14 & $47 \%$ \\
Total & $\mathbf{3 0}$ & $\mathbf{1 0 0}$ & $\mathbf{3 0}$ & $\mathbf{1 0 0}$ \\
\hline
\end{tabular}

Fuente: Pre y post test aplicado a estudiantes IV ciclo - IE 88027

Interpretación: De acuerdo a los datos obtenidos del pre test, se alcanzó evidenciar que el mayor porcentaje de estudiantes se ubicaron en el nivel malo de conciencia ambiental, representado con el 57\%, mientras que en el nivel regular estuvo conformada por el 33\%, sin embargo, los porcentajes más bajos que se manifestaron fueron en el nivel bueno con el $7 \%$ y solamente con el 3\% en el nivel muy bueno. Posterior a la aplicación del programa y recogiendo información con el post test se apreció la predominancia de la cantidad de estudiantes ubicados en el nivel bueno con el 50\%, asimismo, se logró obtener el $47 \%$ en el nivel muy bueno, solo el 3\% se logró ubicar en el nivel regular y ningún estudiante se mantuvo en el nivel malo de conciencia ambiental.

\section{Tabla 2}

Niveles de Conciencia Ambiental por dimensiones

\begin{tabular}{|c|c|c|c|c|c|c|c|c|c|c|c|c|c|c|c|c|}
\hline \multirow{3}{*}{ Nivel } & \multicolumn{4}{|c|}{ COGNITIVA } & \multicolumn{4}{|c|}{ AFECTIVA } & \multicolumn{4}{|c|}{ CONATIVA } & \multicolumn{4}{|c|}{ ACTIVA } \\
\hline & \multicolumn{2}{|c|}{ Pre Test } & \multicolumn{2}{|c|}{ Post Test } & \multicolumn{2}{|c|}{ Pre Test } & \multicolumn{2}{|c|}{ Post Test } & \multicolumn{2}{|c|}{ Pre Test } & \multicolumn{2}{|c|}{ Post Test } & \multicolumn{2}{|c|}{ Pre Test } & \multicolumn{2}{|c|}{ Post Tes } \\
\hline & fi & $\%$ & fi & $\%$ & fi & $\%$ & fi & $\%$ & fi & $\%$ & fi & $\%$ & fi & $\%$ & fi & $\%$ \\
\hline Tolo & 6 & 20 & 0 & 0 & 17 & 57 & & 7 & 7 & 23 & 0 & & 7 & 23 & & 0 \\
\hline 1 & 19 & 63 & 0 & 0 & 7 & 23 & & 13 & 13 & 43 & & 10 & 15 & 50 & & 7 \\
\hline & 5 & 17 & 12 & 40 & 5 & 17 & 12 & 40 & 9 & 30 & 10 & 33 & 8 & 27 & 8 & 27 \\
\hline 17ruy & 0 & 0 & 18 & 60 & 1 & 3 & 12 & 40 & 1 & 3 & 17 & 57 & 0 & 0 & 20 & 67 \\
\hline Total & 30 & 100 & 30 & 100 & 30 & 100 & 30 & 100 & 30 & 100 & 30 & 100 & 30 & 100 & 30 & 100 \\
\hline
\end{tabular}

Fuente: Pre y post test aplicado a estudiantes IV ciclo - IE 88027 
Interpretación: En la dimensión cognitiva se logró observar un incremento de $60 \%$ en el nivel muy bueno y un $23 \%$ en el nivel bueno, destacando que ningún estudiante logró posesionarme en el nivel malo. En la dimensión afectiva se evidenció un incremento de $37 \%$ en el nivel muy bueno y un $23 \%$ en el nivel bueno, destacando una disminución de $10 \%$ en el nivel regular y $50 \%$ en malo. En la dimensión conativa se observó un incremento de $54 \%$ en el nivel muy bueno y un $3 \%$ en el nivel bueno, apreciando una disminución de 33\% en el nivel regular y 23\% en el malo. En la dimensión activa rescató un incremento de $67 \%$ en el nivel muy bueno, destacando que ningún estudiante logró posesionarme en el nivel malo.

\subsection{Análisis inferencial}

\section{Tabla 3}

Prueba de hipótesis del pre test y post test de conciencia ambiental

\begin{tabular}{lc}
\hline & $\begin{array}{c}\text { Variable Conciencia Ambiental } \\
\text { Post Test - Pre Test }\end{array}$ \\
\hline $\mathrm{Z}$ & $-4,800$ \\
Sig. asintótica (bilateral) &, 000 \\
\hline
\end{tabular}

Fuente: Data del pre - post test de Conciencia Ambiental

Interpretación: Los resultados presentados en la Tabla 3, demostraron una diferencia significativa de los datos obtenidos en las dimensiones del post test con respecto del pre test empleados para la presente investigación, evidenciando de manera general el valor de $\mathrm{Z}=-4.800$ inferior a -1.96 y Sig. = 0.000 menor a 0.05 , rechazándose la hipótesis nula y aceptando la hipótesis del investigador, el cual determinó la alta significatividad de la aplicación del programa Produciendo Textos influenciando de manera positiva en la conciencia ambiental de los estudiantes del IV ciclo de educación primaria de la IE $\mathrm{N}^{\circ}$ 88027, con niveles de confianza del 95\%.

\section{DISCUSIÓN}

Teniendo en cuenta el objetivo general con respecto a la variable conciencia ambiental, y de acuerdo a los resultados presentados en la tabla 1, encontramos resultamos similares con la investigación de Guerrero (2020), quien determinó que antes de aplicar algún tipo de programa en los individuos estudiados, se dan niveles elevados de inicio y proceso respecto de su conciencia ambiental. Esto nos hace percibir que, si no logramos contactar desde un primer momento con los individuos desde tempranas edades, su sensibilidad referida al medio ambiente no estará en un desarrollo idóneo, así como nos presentó Rueda (2017) en sus hallazgos en las que determinó que existe carencia en cuanto a la 
formación de conciencia ambiental en la institución educativa $\mathrm{N}^{\circ} 10214$ "Abel Ramos Purihuamán" ya que no se han logrado aplicar propuestas algunas para mejorar la conciencia ambiental en cada uno de los entes de la comunidad educativa.

Del mismo modo se guarda relación con la investigación de Salazar (2017), quien señaló que antes de aplicar su programa observó que tanto el grupo experimental y el grupo control obtuvieron similares niveles de conciencia ambiental. Estas afirmaciones nos hacen tomar en cuenta lo que en un principio ya se ha señalado, sin alguna actuación para el incremento de conciencia ambiental no va haber un buen desenvolvimiento de esta en cada uno de los individuos. En consecuencia, se proponen acciones para mejorar la conciencia ambiental, así como plantea Rueda (2017), quien procedió a diseñar y aplicar una propuesta en Educación Ambiental, teniendo como finalidad mejorar el medio ambiente de la comunidad. De igual manera, siguiendo los resultados de Palma (2020) señaló que en los distintos niveles de educación existe una insuficiente formación de conciencia ecológica, siendo los docentes responsables de debilitar estas acciones negativas. En este mismo camino encontramos la investigación de Suarez (2021) quien a través de los resultados conseguidos en el post test estableció la significatividad de la aplicación del programa propuesto en la investigación, donde observó un incremento de la conciencia ambiental en estudiantes de secundaria. Diferenciándose en el nivel donde se logró aplicar, pero con los mismos resultados sobre el desarrollo de la conciencia ambiental. Igual conclusión se manifiesta en la investigación realizada por Gómez (2017), destacando la influencia positiva a través de la aplicación de su programa, sin embargo, esta variable estuvo representado por otras dimensiones distintas a la presente investigación, las cuales fueron; conocimiento ambiental, posicionamiento ambiental y comportamiento ambiental. De acuerdo a estos resultados presentados se exhibe la conciencia ambiental a través de otras dimensiones, pero lo que se buscó fue el desarrollo de manera general de la conciencia ambiental, es decir, se sigue tomando un solo fin la cual es mejorar la conciencia ambiental en los individuos. Este interés común que se comparte en las investigaciones se sustenta de acuerdo a Avellaneda et al. (2018), como llegar a ser conscientes de los problemas ambientales que se suscitan. Es decir, tomar conciencia de la situación en la que nos encontramos, particularmente del tema ambiental y el desarrollo sostenible, proyectándonos a una armónica vida compartida con la naturaleza. 
Como se ha logrado saber en líneas anteriores el grupo de estudio inicialmente y de acuerdo al diagnóstico realizado no presentaba niveles adecuados de conciencia ambiental, sin embargo, tras trabajar un conjunto de actividades a través del programa produciendo textos se logró determinar un nivel adecuado de conciencia ambiental. Podemos precisar con lo señalado anteriormente que un individuo empieza a moldear su conciencia para poder actuar sobre la naturaleza, ya que en principio no estaba desarrollada, es decir, y tomando en referencia a Du et al. (2018), asimilamos la capacidad de tomar conciencia de los problemas ambientales, interiorizándolos, de tal manera que nos sentimos en armonía con el medio ambiente.

En referencia a la tabla 3, se evidenció los resultados logrados en la contrastación de hipótesis que demostraron la efectividad de la variable experimental, programa produciendo textos, sobre la variable dependiente, conciencia ambiental, asumiendo que las sesiones aplicadas en el programa de la presente investigación ayudaron a desarrollar la conciencia ambiental en los estudiantes del IV ciclo de educación primaria de la IE $\mathrm{N}^{\circ}$ 88027. No obstante, queda resaltar la importancia de seguir de manera oportuna cada una de las actividades previstas en la organización del programa para poder obtener los resultados previstos inicialmente. Estos resultados tienen relación a los obtenidos por Iglesias (2021), quien señaló la obtención de un nivel de significancia $\mathrm{p}=0,000$, determinando la influencia del programa planteado en la investigación sobre el desarrollo de la conciencia ambiental. Asimismo, en la investigación de Ruíz (2019), se determinó que la aplicación de su programa permitió que los estudiantes del $3^{\circ}$ de primaria mejoren la conciencia ambiental en niveles buenos y muy buenos, determinado con una significatividad Sig. < 0,05 y justamente coincidiendo con estos estudios y de acuerdo a Nazarenko y Kolesnik (2018), la conciencia ambiental toma una centralidad en la etapa estudiantil, línea por la cual se da la trascendencia de la competencia ambiental.

En la tabla 2, se presentó los resultados sobre la influencia del programa produciendo textos en el desarrollo de cada una de las dimensiones de la conciencia ambiental, encontrándose sobre la dimensión cognitiva resultados significativos las cuales se afianzan con los aportes de Laso et al. (2019), quienes consideran que la conciencia ambiental se relaciona con la consecución conocimientos que abarcan la problemática ambiental, información exacta con respecto a lo que se está buscando y la emisión de un juicio a partir de ello. Se presenta entonces, que los individuos deben tener el conocimiento adecuado sobre el tema que se piensa abordar para tratarla con mayor 
oportunismo. Es importante entonces que para poder tener un adecuado nivel de conciencia ambiental es imprescindible trabajar el aspecto cognitivo. Con respecto a la dimensión afectiva, se registró un aumento significativo en el post test, logrando coincidir con los estudios de Suarez (2021) quienes mostraron un desarrollo significativo de la conciencia ambiental en la dimensión afectiva. Ante lo presentado, Mediavilla et al. (2020), aportan que la asociación de sentimientos y emociones ayudan directamente con la problemática ambiental, implicando la integración de valores. Sobre la dimensión conativa, se logró un cambio beneficioso luego de aplicar el programa produciendo textos, respaldándonos en López (2020), quien señala que abarca las propias actitudes con su compromiso con el medio ambiente. Sin embargo, en la investigación de García (2019) se evidenció un nivel inferior referida a la dimensión conativa, concluyendo que el accionar de las personas no se percibe adecuadamente, ya que quedan estancados en el proceso de reflexión y discurso. Podemos señalar de acuerdo a los resultados previstos en las distintas investigaciones que es importante para poder tener un adecuado nivel de conciencia ambiental la imprescindibilidad de trabajar el aspecto conativo. En cuanto a la dimensión activa, se evidenció un cambio significativo con los resultados obtenidos en el post test, coincidiendo con los estudios de Salazar (2017) y Suarez (2021) quienes mostraron en sus conclusiones un desarrollo significativo de la conciencia ambiental en la dimensión activa. Al respeto López (2020) manifiesta que es una práctica que evoca la parte individual y colectiva que se proyecta mirando el objetivo que es el cuidado del medio ambiente.

\section{CONCLUSIÓN O CONSIDERACIONES FINALES}

Se concluyó que el programa produciendo textos influye de manera significativa en el desarrollo de la conciencia ambiental de los estudiantes del IV ciclo de educación primaria, como se pudo apreciar en la prueba estadística de Wilcoxon para muestras relacionadas una significancia (Sig.) $=0,000$ y un valor de $Z=-4.800$, rechazando la hipótesis nula y como consecuencia aceptando la hipótesis del investigador.

Se concluyó que a través de la aplicación del programa produciendo textos existe una mejora significativa en la dimensión cognitiva de la conciencia ambiental, obteniéndose un incremento de $23 \%$ en el nivel bueno y un $60 \%$ en el nivel muy bueno. Haciendo referencia que los estudiantes muestran mejores ideas sobre el medio ambiente del cual forman parte. 
Se concluyó que existe una influencia significativa en la dimensión afectiva de la conciencia ambiental, denotándose un aumento de $23 \%$ en el nivel bueno y un $37 \%$ en el nivel muy bueno. Estableciendo que los estudiantes manifiestan mejor su percepción sobre el medio ambiente, valorando su vinculación.

Se concluyó que existe un desarrollo significativo de la dimensión conativa de la conciencia ambiental, alcanzándose un crecimiento de $3 \%$ en el nivel bueno y un $56 \%$ en el nivel muy bueno. Denotándose que los estudiantes tienen mejor disposición para relacionarse con el ambiente, apostando por un desarrollo sostenible.

Se concluyó que después de la aplicación del programa produciendo textos existe un cambio significativo en la dimensión activa de la conciencia ambiental, evidenciándose un crecimiento de $67 \%$ en el nivel muy bueno. Denotándose que los estudiantes mejoraron sus comportamientos y su participación en acciones, de manera responsable, con respecto al cuidado del ambiente que les rodea.

Se elaboró la propuesta de investigación teniendo en cuenta la significatividad del programa produciendo textos para desarrollar la conciencia ambiental en estudiantes de educación primaria, atendiendo la necesidad de mejorar la interrelación del ser humano con el medio ambiente, involucrado la participación de los docentes como guías en este proceso.

\section{LISTA DE REFERENCIAS}

Aliman, M., Budijanto, B., Sumarmi, S. y Astina, I. (2019). Improving Environmental Awareness of High School Students' in Malang City through Earthcomm Learning in the Geography Class. International Journal of Instruction, 12(4), 7994. doi: 10.29333/IJI.2019.1246A

Aparecida, F., Siqueira, A. y De Cássia, R. (2018). Concepções dos Docentes de Engenharia sobre Educação Ambiental. Revista de Ciencia y Tecnología, (29), 27-34. Recuperado de http://www.scielo.org.ar/scielo.php?script=sci_arttext\&pid=S1851$75872018000100009 \& \operatorname{lng}=\mathrm{es} \& \operatorname{lng}=\mathrm{pt}$.

Avellaneda, L., González, A. y Sáez, F. (2018). The Pursuit of External Knowledge in Eco-Innovation. Analysis of the Agri-Food Sector in Spain. Journal of Business, 10(1), 70-92. doi: https://doi.org/https://doi.org/10.21678/jb.2018.878

Banco Mundial (02 de julio de 2020). Contaminación atmosférica: confinada pero no detenida por la COVID-19. Recuperado de 
https://www.bancomundial.org/es/news/immersive-story/2020/07/01/airpollution-locked-down-by-covid-19-but-not-arrested

Castillo, M., Ruíz, C. y Flores, K. (2018). La auditoría ambiental como instrumento de prevención de la contaminación en las empresas laminadoras, Pucallpa 2014. Revista de Investigación Científica Cultura Viva Amazónica, 3(3), 25-31. doi: https://doi.org/10.37292/riccva.v3i03.117

Chierrito, E., Martins, A., De Souza, E., Da Silva, R. y Grossi, R. (2018). Proenvironmental behavior and recycling: literature review and policy considerations. Ambiente \& Sociedade, 21(11). doi: https://dx.doi.org/10.1590/1809-4422asoc0209r3vu1814ao

Di Alessio, B. y Destefanis, L. (2017). El desarrollo de la capacidad de producción escrita en la escuela secundaria. Cuadernillo para profesores. Educación para todos Asociación civil. Argentina.

Du, Y., Wang, X., Brombal, D., Moriggi, A., Sharpley, A. y Pang, S. (2018). Changes in Environmental Awareness and Its Connection to Local Environmental Management in Water Conservation Zones: The Case of Beijing, China. Sustainability, 10(6), 1-24. doi: https://doi.org/10.3390/su10062087

Eliseo, P. (2020). Navigating Environmental Education Practices to Promote Environmental Awareness and Education. International Journal on Studies in Education, 2(1), 45-57. Recuperado de https://eric.ed.gov/?q=environmental+awareness\&pg=2\&id=ED607231.

Emine, T. (2019). Teacher Canditates' Environmental Awareness and Environmental Sensitivity. International Journal of Higher Education 8(4). 202-207. doi: https://doi.org/10.5430/ijhe.v8n4p202

Falconí, F., Reinoso, M., Collado, J., Hidalgo, E. y León, G. (2019). Environmental education program in Ecuador: theory, practice, and public policies to face global change in the Anthropocene. Ensaio: Avaliação e Políticas Públicas em Educação, 27(105), $\quad$ 859-880. doi: https://doi.org/10.1590/s010440362019002701950

Flores, P., Gómez, N. y Osman, J. (2017). Urbanismo e infancia: hacia un modelo de ciudad que promueva la conciencia ambiental. Chasqui. Revista Latinoamericana de Comunicación, (136), 81-96. Recuperado de https://revistachasqui.org/index.php/chasqui/article/view/3305 
García, N. (2019). PERCEPCIÓN CIUDADANA DE LOS PROBLEMAS MEDIOAMBIENTALES Y DE SOSTENIBILIDAD URBANA EN CONTEXTOS METROPOLITANOS Análisis desde las agendas 21 de cuatro municipios de la comunidad de Madrid (España) (Tesis doctoral). Universidad Nacional de Educación a Distancia. España.

Gervacio, H. y Castillo, B. (2020). Conocimientos, actitudes y prácticas socioambientales en estudiantes de la Universidad Autónoma de Guerrero, México. RIDE. Revista Iberoamericana para la Investigación y el Desarrollo Educativo, 11(21), e057. Recuperado de http://www.scielo.org.mx/scielo.php?script=sci_arttext\&pid=S2007$74672020000200157 \& \operatorname{lng}=$ es.

Gómez, L. (2017). Programa experimental para fomentar la conciencia ambiental en estudiantes del quinto grado de primaria de la I.E. $N^{o} 11016$ del Pueblo Joven José Olaya, Chiclayo (Tesis doctoral). Universidad césar Vallejo. Chiclayo, Perú.

González, E., y Meira, P. (2020). Educación para el cambio climático: ¿Educar sobre el clima o para el cambio?. Perfiles educativos, 42(168), 157-174. doi: https://doi.org/10.22201/iisue.24486167e.2020.168.59464

Guerrero, H. (2020). Propuesta de Manejo de Residuos Sólidos para Mejorar la Conciencia Ambiental en los Estudiantes de quinto y sexto grado de la Institución Pública Canchaque 2020 (Tesis doctoral). Universidad César Vallejo. Piura, Perú.

Iglesias, J. (2021). Programa Ambienti Kids en la conciencia ambiental de estudiantes del tercer grado de primaria, Institución Educativa Manuel María Álvarez, Cascas - 2020 (Tesis doctoral). Universidad César Vallejo. Trujillo, Perú.

Instituto Nacional de Estadística e informática. (2021). Informe técnico Estadísticas Ambientales (informe $\quad \mathrm{N}^{\circ}$ 05). Recuperado de https://www.inei.gob.pe/media/MenuRecursivo/boletines/05-informe-tecnicoestadisticas-ambientales-abr-2021.pdf

Kabadayi A. y Altinsoy, F. (2019). Traditional and Technological Methods for Raising Pre-school Children's Awareness of Environmental Pollution for Sustainability. Discourse and Communication for Sustainable Education, 9(2), 134-144. doi: https://doi.org/10.2478/dcse-2018-0020 
Laso, S., Marban, J. y Ruíz, M. (2017). Environmental Awareness as a Mediator Variable on Conceptions of Science Teaching and Learning. International journal of environmental \& science education, 12(5), 1401-1426. Recuperado de https://eric.ed.gov/?q=environmental+awareness\&pg=2\&id=EJ1278146

Laso, S., Marbán, J. y Ruiz, M. (2019). Impacto de un programa de intervención metacognitivo sobre la Conciencia Ambiental de docentes de Primaria en formación inicial. Revista Eureka sobre Enseñanza y Divulgación de las Ciencias 16(2), 2501. Recuperado de https://www.semanticscholar.org/paper/Impacto-deun-programa-de-intervenci\%C3\%B3n-sobre-la-de-SalvadorPastrana/3742770dcb7f463c3de2e75e046405dc0f3f61b6

León, Y., Gomera, A., Antúnez, M., Martínez, B., Villamandos, F. y Vaquero, M. (2018). Enhancing environmental management in universities through participation: the case of the University of Córdoba. Journal of Cleaner Production, 172, 43284337. Doi: https://doi.org/10.1016/j.jclepro.2017.06.103.

López, Y. (2020). Proyecto educativo ambiental integrado y el desarrollo de la conciencia ambiental en los estudiantes del IESTP Antenor Orrego Espinoza (Tesis doctoral). Universidad Nacional Federico Villarreal. Lima. Perú.

Mediavilla, M., Medina, S. y González, I. (2020). Diagnóstico de sensibilidad medioambiental en estudiantes universitarios. Educación y Educadores, 23(2), 179-197. doi: https://doi.org/10.5294/edu.2020.23.2.2

Ministerio de educación (2015). ¿Qué y cómo aprenden nuestros estudiantes? Comunicación. Lima. Perú.

Ministerio de Educación. (2017). Currículo Nacional de Educación Básica. Lima. Perú.

Moyano, E. (2018). Un ensayo sobre la Laudato si' y su contribución a la conciencia ambiental. Revista de Fomento Social, 73(291), 441-456. Recuperado de https://www.revistadefomentosocial.es/rfs/article/view/1512

Nagihan, Ö. (2020). Investigation of gifted students' environmental awareness. International Journal of Curriculum and Instruction, 12(2), 95-107. Recuperado de https://eric.ed.gov/?q=environmental+awareness\&id=EJ1271109

Nazarenko, A. y Kolesnik, A. (2018). Raising Environmental Awareness of Future Teachers. International Journal of Instruction, 11 (3), 63-76. Recuperado de https://eric.ed.gov/?id=EJ1183379 
Organización Mundial de la Salud. (2018). Importante acuerdo de colaboración entre el ONU Medio Ambiente y la OMS sobre los riesgos sanitarios de origen ambiental. Recuperado de https://www.who.int/es/news/item/10-01-2018-un-environmentand-who-agree-to-major-collaboration-on-environmental-health-risks

Ospina, J. (2019). Las capitales y países de América Latina más contaminados. Recuperado de https://p.dw.com/p/3Icp9

Palma, N. (2020). Conciencia ecológica en los docentes de Educación Básica Regular: un análisis hermenéutico desde la realidad educativa (Tesis doctoral). Universidad César Vallejo. Chimbote, Perú.

Punzalan, C., Signo, C., Mae, C., Signo, M. y Marasigan, A. (2019). Environmental Awareness of Selected Urban and Rural High School Students in the Philippines. Journal on School Educational Technology, 15(2), 15-25. doi: https://doi.org/10.26634/jsch.15.2.16664

Quispe, M., Rojas, K., Auccahuasi, W., Urbano, K., Flores, P., y Heredia, W. (2020). Social responsibility as a means of business sustainability, case of a mining company in peru. Investigaciones Sobre Lectura, 17(6), 14676-4684. Recuperado de https://archives.palarch.nl/index.php/jae/article/view/4880

Rathee, N. y Thakran, S. (2017). A Study of environmental awareness among rural and urban secondary school students. International Education and Research, 3(5), 204-205. Recuperado de https://www.semanticscholar.org/paper/A-STUDY-OFENVIRONMENTAL-AWARENESS-AMONG-RURAL-AND-RatheeThakran/8bcc7d0c754bc53849f5885472c79d2327afd60e

Revista Semana. (27 de enero de 2021). Medioambiente. Frenar la deforestación: principal reto ambiental de Colombia en 2021. Recuperado de https://www.semana.com/medio-ambiente/articulo/retos-medioambientales-decolombia-en-2021---noticias-hoy/58951/

Rueda, R. (2017). Programa en educación ambiental para fomentar la conciencia ambiental (Tesis doctoral). Universidad Nacional Pedro Ruiz Gallo. Lambayeque. Perú.

Ruiz, Y. (2019). Programa "Mi Mundo Verde" en el desarrollo de la conciencia ambiental de los estudiantes del tercer grado de primaria de la Institución Educativa 14901, Pariñas - Talara, 2018 (Tesis doctoral). Universidad César Vallejo. Talara. Perú. 
Salazar, A. (2017). Programa "Educamp" en la conciencia ambiental de los estudiantes del nivel primaria, Esperanza, Trujillo-2017 (Tesis Doctoral). Universidad César Vallejo. Trujillo. Perú.

Santacruz, A. (2018). La estrategia del debate en el fortalecimiento de la conciencia ambiental. Investigación Valdizana, 12(4),177-183. Recuperado de https://www.redalyc.org/articulo.oa?id=586062188001

Suárez, T. (2021). Programa "Dejando huellas verdes" para el fortalecimiento de la conciencia ambiental en el área de ciencia y tecnología en educación secundaria (Tesis doctoral). Universidad César Vallejo. Lima. Perú.

Susilawati, Aznam, N., Irwanto, I. y Paidi. (2020). Socio-Scientific Issues as a Vehicle to Promote Soft Skills and Environmental Awareness. European Journal of Educational Research, 10(1), 161-174. doi: https://doi.org/10.12973/eujer.10.1.161

Vargas, R. (2021). Propuesta metodológica para el desarrollo de la conciencia ambiental en estudiantes de la Institución Educativa Los Licenciados de Ayacucho - 2019. Horizonte de la Ciencia, 11(20), 223-233. Recuperado de https://www.redalyc.org/articulo.oa?id=570965027017

Vine, A. (2020). La escritura académica: percepciones de estudiantes de Ciencias Humanas y Ciencias de la Ingeniería de una universidad chilena. Íkala, Revista de Lenguaje $\quad y \quad$ Cultura, 25(2), 475-491. doi: https://doi.org/10.17533/udea.ikala.v25n02a02

Yeşilyurt, M., Özdemir, M. y Erol, M. (2020). The Impact of Environmental Education Activities on Primary School Students' Environmental Awareness and Visual Expressions. Qualitative Research in Education, 9(2), 188-216. doi: 10.17583/qre.2020.5115

Y1ldiz, N. (2019). An examination of the relationship between primary school students' environmental awareness and basic science process skills. Educational Research and Reviews, 14(4), 140- 151. doi: 10.5897/ERR2018.3663 